\title{
Ruthenium-catalyzed cross aldol reaction with aldehydes and ketones
}

\author{
Khalil Tabatabaeian,* Manouchehr Mamaghani, Nosrat O. Mahmoodi, \\ and Elahe Keshavarz \\ Department of Chemistry, Faculty of Science, University of Guilan, Rasht, P.O. Box 41335- \\ 1914, Iran \\ E-mail:taba@guilan.ac.ir
}

\begin{abstract}
Catalytic amounts of ruthenium (II) catalyzed the efficient cross aldol reactions of aryl alkyl ketones with aromatic and heteroaromatic aldehydes without the presence of any dehydrated product. The resulted conditions led to the corresponding aldol products in moderate to good yields. Under microwave irradiation, the reaction conditions were improved.
\end{abstract}

Keywords: Ruthenium, aldehyde, ketone, aldol product

\section{Introduction}

Transition metal-catalyzed (C-C) bond-forming reactions have received considerable attention in organic chemistry. ${ }^{1}$ Much effort has been devoted to develop more convenient and efficient strategies for the formation of $\mathrm{C}-\mathrm{C}$ bonds. The aldol reaction is one of the most powerful methods for the formation of $\mathrm{C}-\mathrm{C}$ bonds in organic synthesis. ${ }^{2-4}$ The aldol products constitute the backbone of many important drugs and other bioactive molecules. The frequent occurrence of a $\beta$-hydroxyl carbonyl moiety in a variety of natural products has stimulated the development of synthetic methods for the preparation of these compounds. However, under the classical aldol reaction conditions, dimerization, polymerization, and self-condensation also occur. ${ }^{5}$ To avoid such competing processes, an important modification of the classical aldol reaction has been developed by treating enol silyl ethers with carbonyl compounds in the presence of Lewis acids ( the Mukaiyama aldol reaction ). ${ }^{6}$

A highly efficient formation of $\beta$-hydroxy ketones via vanadium-catalyzed coupling of propargyl alcohols with aldehydes has been reported. ${ }^{7}$ Recent developments also include hydrometallation-aldol ${ }^{8}$ and $\mathrm{C}$-H activation-aldol reactions of carbonyl compounds. ${ }^{9,}{ }^{10}$ Gree and co-workers recently reported the isomerization of allylic alcohols to enols catalyzed by $\mathrm{Fe}(\mathrm{CO})_{5}$ under photolytic conditions, which then reacted with aldehydes to give aldol products (in 
addition to other products). ${ }^{11}$ Also an elegant chemical method has been developed by Trost which provide aldols from aldehydes in modest yields. ${ }^{12}$

On the other hand, with recent progress in organometallic chemistry, organic synthesis catalyzed by ruthenium compounds has attracted much attention, and a large number of useful catalytic reactions have been discovered. ${ }^{13}$ Here, we report ruthenium (II) as an effective catalyst for the aldol reaction, yielding $\beta$-hydroxy ketones in good yields. In this study, the ruthenium catalyst was used in low concentration and no dehydrated products were produced in all cases.

\section{Results and Discussion}

The study of the aldol reaction is actively pursued in order to improve the reaction conditions and to extend the scope and applicability of this type of reaction. We herein report the $\mathrm{Ru}^{\mathrm{II}}$ efficiently catalyzed intermolecular aldol reaction of aromatic and heteroaromatic aldehydes with aryl alkyl ketones. In this method in contrast to the conventional synthesis which frequently requires high loading or stoichiometric quantities of catalyst, ${ }^{14}$ small amounts of catalyst were used in all cases. Therefore, the reaction not only allows the reduction of waste but also results in the diminution of costs.

It is worth noting that aldol products were stable under the reaction conditions and no reversibility was observed. Also the formation of significant amounts of the aldol condensation product $^{15}$ and aldehyde self-aldolization were completely suppressed. Therefore, in this method, no side products of catalytic aldol reactions were found and the corresponding aldol products were obtained in good yields. Also, the operational simplicity of this protocol and mild reaction conditions, are the advantages of this method to the other methods used for the reaction of aryl alkyl ketones and aldehydes.

The reaction could be extended to some aryl alkyl ketones such as acetophenone (2a), 4methyl acetophenone (2b), propiophenone (2c), and in each case, the aldol products (3aa-3fc) were obtained in moderate to good yields. In the case of (2c), a diastereomeric mixture was obtained ( $d r=43: 57,42: 58,40: 60$ for (3cc), (3dc), (3ec); 47:53 for (3bc) and (3fc) respectively) (entries 7-11 of the Table 1). Typical results of the ruthenium-catalyzed aldol reactions of aromatic and heteroaromatic aldehydes and aryl alkyl ketones, under optimized reaction conditions, are shown in Table 1 . Treatment of aldehydes $(1 \mathrm{mmol})$ with ketones $(1 \mathrm{mmol})$ in the presence of $\mathrm{RuCl}_{2}\left(\mathrm{PPh}_{3}\right)_{3}$ catalyst $(2 \mathrm{~mol} \%)$ and $\mathrm{KOH}(0.8 \mathrm{mmol})$ in dioxane $(2 \mathrm{~mL})$ at $80^{\circ} \mathrm{C}$ gave the corresponding $\beta$-hydroxy ketones in moderate to good yields.

Both aromatic and aliphatic aldehydes were used as the carbonyl partner in the reaction system. In the case of aliphatic aldehydes the reactions were slow and did not readily go to completion. 
Table 1. Ruthenium-catalyzed aldol reaction of aldehydes with ketones

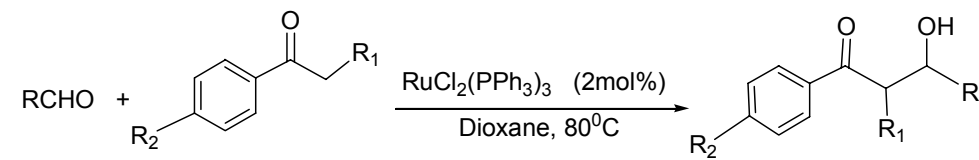

1a-f 2a-c Aldol product

$1 \mathrm{a}: \mathrm{R}=\mathrm{Ph}$

1b: $\mathrm{R}=4-\mathrm{MeSC}_{6} \mathrm{H}_{4}$

1c: $\mathrm{R}=5$-Methylthiophen-2-yl

$1 \mathrm{~d}: \mathrm{R}=5$-Bromothiophen-2-yl

1e: $\mathrm{R}=3$-Methylthiophen-2-yl

1f: $\mathrm{R}=$ Furan-2-yl 2a: $\mathrm{R}^{1}=\mathrm{R}^{2}=\mathrm{H}$

2b: $\mathrm{R}^{1}=\mathrm{H}, \mathrm{R}^{2}=\mathrm{Me}$

2c: $\mathrm{R}^{1}=\mathrm{Me}, \mathrm{R}^{2}=\mathrm{H}$

\begin{tabular}{|c|c|c|c|c|c|c|c|}
\hline \multirow[b]{2}{*}{ Entry $^{\mathrm{a}}$} & \multirow[b]{2}{*}{ Aldehyde } & \multirow[b]{2}{*}{ Ketone } & \multirow[b]{2}{*}{$\begin{array}{c}\text { Aldol } \\
\text { Product }\end{array}$} & \multirow[b]{2}{*}{$\begin{array}{l}\text { Yield } \\
(\%)^{b}\end{array}$} & \multirow[b]{2}{*}{ Time $(\mathrm{h})$} & \multicolumn{2}{|c|}{ Microwave Irradiation } \\
\hline & & & & & & $\begin{array}{l}\text { Yield } \\
(\%)^{b}\end{array}$ & $\begin{array}{l}\text { Time } \\
(\mathrm{min})\end{array}$ \\
\hline 1 & $1 \mathrm{a}$ & $2 a$ & $3 a a$ & 72 & 34 & $70^{c}$ & 1.5 \\
\hline 2 & $1 b$ & $2 \mathrm{a}$ & $3 \mathrm{ba}$ & 75 & 34 & 74 & 1 \\
\hline 3 & $1 b$ & $2 b$ & $3 \mathrm{bb}$ & 78 & 30 & 62 & 1 \\
\hline 4 & $1 \mathrm{c}$ & $2 \mathrm{a}$ & $3 \mathrm{ca}$ & 55 & 40 & 75 & 2 \\
\hline 5 & $1 d$ & $2 b$ & $3 \mathrm{db}$ & 81 & 20 & 91 & 1.5 \\
\hline 6 & $1 \mathrm{e}$ & $2 b$ & $3 e b$ & 69 & 18 & 75 & 1 \\
\hline 7 & $1 b$ & $2 \mathrm{c}$ & $3 b c$ & 72 & 9 & 69 & 1 \\
\hline 8 & $1 \mathrm{c}$ & $2 \mathrm{c}$ & $3 \mathrm{cc}$ & 58 & 10 & 80 & 1.5 \\
\hline 9 & $1 d$ & $2 \mathrm{c}$ & $3 \mathrm{dc}$ & 85 & 4 & 92 & 1 \\
\hline 10 & $1 \mathrm{e}$ & $2 \mathrm{c}$ & $3 e c$ & 67 & 12 & 78 & 2.5 \\
\hline 11 & $1 \mathrm{f}$ & $2 \mathrm{c}$ & $3 \mathrm{fc}$ & 68 & 6 & 68 & 1.5 \\
\hline
\end{tabular}

${ }^{a}$ All products were characterized by ${ }^{1} \mathrm{H}$ NMR, ${ }^{13} \mathrm{C}$ NMR and IR data. ${ }^{b}$ Yields after purification by chromatography. ${ }^{\mathrm{c}}$ Identified by comparison with authentic samples. ${ }^{16}$

A proposed mechanistic pathway for the aldol reaction is presented in Scheme 1. The mechanism involving ruthenium enolates which can be trapped by an aldehyde to give the aldol product, is proposed. In order to rationalize the proposed mechanism, "blank" experiments were tested on substrates $\mathbf{1 b}$ and $\mathbf{1 d}$ (entries 2 and 5 of the Table 1) without using ruthenium catalysis. In all cases, the reaction produced the aldol products in much lower yields and with much longer reaction times, along with dehydrated products and self-condensation. Because of these drawbacks, it should be the cooperation of catalyst and base, which led to the effective catalytic activity of our reaction system. 


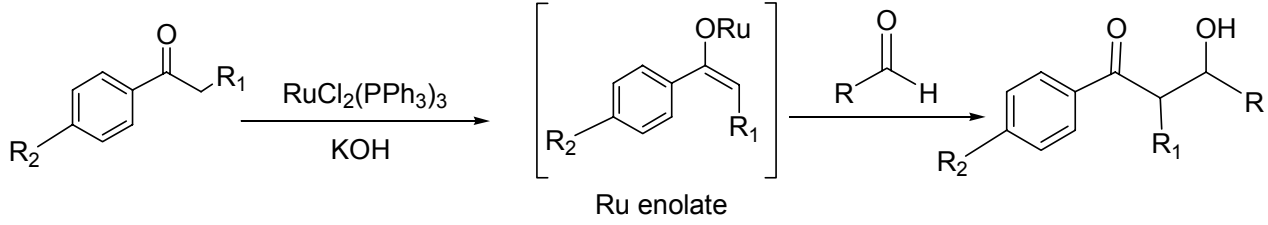

\section{Scheme 1}

Finally, as shown in Table 1, we decided to overcome the problem of somehow prolonged reaction times using microwave irradiation. A mixture of aldehydes $(1 \mathrm{mmol})$, ketones $(1 \mathrm{mmol})$, base $(0.8 \mathrm{mmol})$ and $2 \mathrm{~mol} \%$ of the catalyst was dissolved in the minimum amount of solvent and irradiated in a $160 \mathrm{~W}$ microwave oven in an open vessel and the progress of the reaction was monitored by TLC. After the indicated reaction time (1-2.5 min), the crude mixture was purified by preparative TLC (12:3, petroleum ether/ethyl acetate) and provided the desired products. Generally, the products were produced without the formation of dehydrated products. These results revealed the potential application of microwave irradiation in the promotion of the catalyzed reaction.

In conclusion, in continuation of our recent work on the catalytic reactivity of ruthenium, ${ }^{17}$ this procedure extends the methodology of ruthenium-catalyzed aldol reactions including reactions of aldehydes and aryl alkyl ketones. The cooperation of catalyst and base, which led to the effective catalytic activity of our reaction system is believed to be the key of the reaction. To the best of our knowledge, this is the first report on the formation of aldol adducts with heteroaromatic aldehydes using ruthenium (II) as catalyst. The ruthenium seems an efficient catalyst since as little as $2 \mathrm{~mol} \%$ is enough to efficiently catalyze cross aldol reaction. In this method, the catalyst is easy to prepare and the reaction occurs under mild conditions. Also, the operational simplicity of this protocol, the ease of work-up and the small amount of waste are advantages of this protocol. More importantly, there is no dehydrated product produced in all cases. However, diastereoselectivity toward one diastereoisomer remains a challenging object. Further investigations focusing on the full scope of this method are currently in progress in our laboratory.

\section{Experimental Section}

General Procedures. IR spectra were recorded on Shimadzu FTIR-8400S spectrometer. ${ }^{1} \mathrm{H}$ NMR spectra were obtained on a Bruker DRX-500 Avance spectrometer and ${ }^{13} \mathrm{C}$ NMR were obtained on a Bruker DRX-125 Avance spectrometer. Samples were analyzed in $\mathrm{CDCl}_{3}$, and the chemical shift values are expressed relative to $\mathrm{Me}_{4} \mathrm{Si}$ as an internal standard. Elemental analyses were made by a Carlo-Erba EA1110 CNNO-S analyzer and agreed with the calculated values. The isolation of pure products was carried out via preparative thin layer chromatography (Silica Gel $60 \mathrm{GF}_{254}$; Merck). $\mathrm{RuCl}_{2}\left(\mathrm{PPh}_{3}\right)_{3}$ was prepared as described in the literature. ${ }^{18}$ Solvents, 
organic and inorganic compounds were purchased from Merck and used without further purification.

Typical method. To a solution of the aldehyde $(1 \mathrm{mmol})$ and ketone $(1 \mathrm{mmol})$ in dioxane $(2$ $\mathrm{mL}), \mathrm{RuCl}_{2}\left(\mathrm{PPh}_{3}\right)_{3}(19.0 \mathrm{mg}, 0.02 \mathrm{mmol})$ and $\mathrm{KOH}(0.8 \mathrm{mmol})$ were added. The resulting mixture was allowed to react at $80 \mathrm{C}$ and monitored by TLC. After the indicated reaction time (Table 1), the reaction mixture was cooled to room temperature and purified by thin layer chromatography on Silica Gel (petroleum ether/ethyl acetate 12:3) to give the corresponding aldol. For microwave reactions, irradiation was conducted in an open vessel in a $160 \mathrm{~W}$ microwave oven.

3-Hydroxy-3-(4-(methylthio)phenyl)-1-phenylpropan-1-one (3ba). A colorless oil, IR (neat): v $\left(\mathrm{cm}^{-1}\right): 3508,3075,2958,1726,1679,1595,1492 .{ }^{1} \mathrm{H} \mathrm{NMR}\left(500 \mathrm{MHz}, \mathrm{CDCl}_{3}, 25^{\circ} \mathrm{C}\right): \delta=2.58$ $(\mathrm{s}, 3 \mathrm{H}), 3.41(\mathrm{~d}, \mathrm{~J}=6.0 \mathrm{~Hz}, 2 \mathrm{H}), 3.61(\mathrm{~d}, \mathrm{~J}=2.9 \mathrm{~Hz}, \mathrm{OH}), 5.36(\mathrm{~m}, 1 \mathrm{H}), 7.33(\mathrm{~m}, 2 \mathrm{H}), 7.42(\mathrm{~m}$, 2H), $7.53(\mathrm{~m}, 2 \mathrm{H}), 7.65(\mathrm{~m}, 1 \mathrm{H}), 8.00(\mathrm{~m}, 2 \mathrm{H}) \mathrm{ppm} .{ }^{13} \mathrm{C} \mathrm{NMR}\left(125 \mathrm{MHz}, \mathrm{CDCl}_{3}, 25^{\circ} \mathrm{C}\right): \delta=$ 46.2 , 52.5, 68.1, 113.5, 126.1, 126.5, 128.0, 129.3, 135.2, 136.5, 160.5, 200.2 ppm. Anal. Calcd for $\mathrm{C}_{16} \mathrm{H}_{16} \mathrm{O}_{2} \mathrm{~S}: \mathrm{C}, 70.58 ; \mathrm{H}, 5.88 ; \mathrm{S}, 11.76$; found: C, 70.59; H, 5.87; S, 11.79.

3-Hydroxy-3-(4-(methylthio)phenyl)-1-p-tolylpropan-1-one (3bb). A colorless oil, IR (neat): v $\left(\mathrm{cm}^{-1}\right): 3487,3042,2925,1726,1674,1604,1458 .{ }^{1} \mathrm{H}$ NMR $\left(500 \mathrm{MHz}, \mathrm{CDCl}_{3}, 25^{\circ} \mathrm{C}\right): \delta=$ $2.46(\mathrm{~s}, 3 \mathrm{H}), 2.53(\mathrm{~s}, 3 \mathrm{H}), 3.38(\mathrm{~m}, 2 \mathrm{H}), 3.69(\mathrm{~d}, \mathrm{~J}=2.7 \mathrm{~Hz}, \mathrm{OH}), 5.34(\mathrm{~m}, 1 \mathrm{H}), 7.32(\mathrm{~m}, 2 \mathrm{H})$, $7.41(\mathrm{~m}, 2 \mathrm{H}), 7.59(\mathrm{~m}, 1 \mathrm{H}), 7.76(\mathrm{~m}, 1 \mathrm{H}), 7.95(\mathrm{~m}, 2 \mathrm{H}) \mathrm{ppm} .{ }^{13} \mathrm{C}$ NMR $\left(125 \mathrm{MHz}, \mathrm{CDCl}_{3}\right.$, $\left.25^{\circ} \mathrm{C}\right): \delta=47.2,50.1,52.1,69.1,113.5,126.2,126.5,128.0,128.3,133.2,136.5,158.6,200.1$ ppm. Anal. Calcd for $\mathrm{C}_{17} \mathrm{H}_{18} \mathrm{O}_{2} \mathrm{~S}$ : C, 71.32; H, 6.29; S, 11.18; found: C, 71.35; H, 6.31; S, 11.17. 3-Hydroxy-3-(5-methylthiophen-2-yl)-1-phenylpropan-1-one (3ca). A colorless oil, IR (neat): v $\left(\mathrm{cm}^{-1}\right): 3450,3060,2920,1730,1635,1460 .{ }^{1} \mathrm{H} \mathrm{NMR}\left(500 \mathrm{MHz}, \mathrm{CDCl}_{3}, 25^{\circ} \mathrm{C}\right): \delta=2.52(\mathrm{~s}$, $3 \mathrm{H}), 3.39(\mathrm{~m}, 2 \mathrm{H}), 3.64(\mathrm{~d}, \mathrm{~J}=3.2 \mathrm{~Hz}, \mathrm{OH}), 5.41(\mathrm{~m}, 1 \mathrm{H}), 6.83(\mathrm{~m}, 2 \mathrm{H}), 6.71(\mathrm{~d}, \mathrm{~J}=3.2 \mathrm{~Hz}$, 1H), $7.60(\mathrm{~m}, 2 \mathrm{H}), 7.99(\mathrm{~d}, \mathrm{~J}=7.4 \mathrm{~Hz}, 2 \mathrm{H}) \mathrm{ppm} .{ }^{13} \mathrm{C} \mathrm{NMR}\left(125 \mathrm{MHz}, \mathrm{CDCl}_{3}, 25^{\circ} \mathrm{C}\right): \delta=21.5$, 46.6, 65.9, 125.4, 128.3, 128.7, 128.9, 129.2, 134.1, 135.1, 137.8, 200.0 ppm. Anal. Calcd for $\mathrm{C}_{14} \mathrm{H}_{14} \mathrm{O}_{2} \mathrm{~S}: \mathrm{C}, 68.29 ; \mathrm{H}, 5.69$; S, 13.00; found: $\mathrm{C}, 68.31 ; \mathrm{H}, 5.71 ; \mathrm{S}, 12.90$.

3-Hydroxy-3-(5-bromothiophen-2-yl)-1-p-tolylpropan-1-one (3db). A colorless oil, IR (neat): $v\left(\mathrm{~cm}^{-1}\right): 3504,3065,2929,1739,1660,1604,1463 .{ }^{1} \mathrm{H} \mathrm{NMR}\left(500 \mathrm{MHz}, \mathrm{CDCl}_{3}, 25^{\circ} \mathrm{C}\right): \delta=$ $2.47(\mathrm{~s}, 3 \mathrm{H}), 3.48(\mathrm{~m}, 2 \mathrm{H}), 3.87(\mathrm{~d}, \mathrm{~J}=3.6 \mathrm{~Hz}, \mathrm{OH}), 5.53(\mathrm{~m}, 1 \mathrm{H}), 6.81(\mathrm{~d}, \mathrm{~J}=3.6 \mathrm{~Hz}, 1 \mathrm{H}), 6.97$ $(\mathrm{d}, \mathrm{J}=3.7 \mathrm{~Hz}, 1 \mathrm{H}), 7.30(\mathrm{~d}, \mathrm{~J}=8.1 \mathrm{~Hz}, 2 \mathrm{H}), 7.91(\mathrm{~d}, \mathrm{~J}=8.1 \mathrm{~Hz}, 2 \mathrm{H}) \mathrm{ppm} .{ }^{13} \mathrm{C} \mathrm{NMR}(125 \mathrm{MHz}$, $\left.\mathrm{CDCl}_{3}, 25^{\circ} \mathrm{C}\right): \delta=22.3,46.8,71.5,125.6,126.1,128.6,128.9,129.6,133.4,134.7,138.5,200.2$ ppm. Anal. Calcd for $\mathrm{C}_{14} \mathrm{H}_{13} \mathrm{O}_{2} \mathrm{SBr}$ C, 51.69; H, 4.00; S, 9.84; found: C, 51.70; H, 3.98; S, 9.85. 3-Hydroxy-3-(3-methylthiophen-2-yl)-1-p-tolylpropan-1-one (3eb). A colorless oil, IR (neat): v $\left(\mathrm{cm}^{-1}\right): 3421,3045,2921,1674,1606,1404 .{ }^{1} \mathrm{HNMR}\left(500 \mathrm{MHz}, \mathrm{CDCl}_{3}, 25^{\circ} \mathrm{C}\right): \delta=2.30(\mathrm{~s}$, $3 \mathrm{H}), 2.47(\mathrm{~s}, 3 \mathrm{H}), 3.51(\mathrm{~m}, 2 \mathrm{H}), 3.71(\mathrm{~d}, \mathrm{~J}=2.5 \mathrm{~Hz}, \mathrm{OH}), 5.68(\mathrm{~m}, 1 \mathrm{H}), 6.89(\mathrm{~d}, \mathrm{~J}=5.0 \mathrm{~Hz}, 1 \mathrm{H})$, $7.23(\mathrm{~d}, \mathrm{~J}=5.0 \mathrm{~Hz}, 1 \mathrm{H}), 7.33(\mathrm{~m}, 2 \mathrm{H}), 7.92(\mathrm{~d}, \mathrm{~J}=8.0 \mathrm{~Hz}, 2 \mathrm{H}) \mathrm{ppm} .{ }^{13} \mathrm{CNMR}(125 \mathrm{MHz}$, $\left.\mathrm{CDCl}_{3}, 25^{\circ} \mathrm{C}\right): \delta=20.9,22.3,50.1,77.6,125.8,128.1,128.3,128.7,130.9,134.2,136.2,139.2$, 
200.3 ppm. Anal. Calcd for $\mathrm{C}_{15} \mathrm{H}_{16} \mathrm{O}_{2} \mathrm{~S}: \mathrm{C}, 69.23 ; \mathrm{H}, 6.15 ; \mathrm{S}, 12.30$; found: $\mathrm{C}, 69.25 ; \mathrm{H}, 6.17 ; \mathrm{S}$, 12.32 .

3-Hydroxy-2-methyl-3-(4-(methylthio)phenyl)-1-phenylpropan-1-one (3bc). A colorless oil, IR (neat): $v\left(\mathrm{~cm}^{-1}\right): 3450,3070,2925,1674,1595,1488$. Data for major isomer. ${ }^{1} \mathrm{H}$ NMR (500 $\left.\mathrm{MHz}, \mathrm{CDCl}_{3}, 25^{\circ} \mathrm{C}\right): \delta=1.24(\mathrm{~d}, \mathrm{~J}=7.2 \mathrm{~Hz}, 3 \mathrm{H}), 2.53(\mathrm{~s}, 3 \mathrm{H}), 3.64(\mathrm{~d}, \mathrm{~J}=4.5 \mathrm{~Hz}, \mathrm{OH}), 3.73(\mathrm{~m}$, $1 \mathrm{H}), 5.25(\mathrm{~m}, 1 \mathrm{H}), 7.29(\mathrm{~m}, 2 \mathrm{H}), 7.36(\mathrm{~m}, 2 \mathrm{H}), 7.51(\mathrm{~m}, 2 \mathrm{H}), 7.61(\mathrm{~m}, 1 \mathrm{H}), 7.99(\mathrm{~d}, \mathrm{~J}=7.8 \mathrm{~Hz}$, 2H) ppm. ${ }^{13} \mathrm{C}$ NMR $\left(125 \mathrm{MHz}, \mathrm{CDCl}_{3}, 25^{\circ} \mathrm{C}\right): \delta=12.8,28.7,47.9,53.8,127.8,128.1,128.6$, $128.8,129.8,139.5,139.7,140.4,201.6 \mathrm{ppm}$. Data for minor isomer. ${ }^{1} \mathrm{H}$ NMR $(500 \mathrm{MHz}$, $\left.\mathrm{CDCl}_{3}, 25^{\circ} \mathrm{C}\right): \delta=1.13(\mathrm{~d}, \mathrm{~J}=7.2 \mathrm{~Hz}, 3 \mathrm{H}), 2.53(\mathrm{~s}, 3 \mathrm{H}), 2.99(\mathrm{~d}, \mathrm{~J}=3.4 \mathrm{~Hz}, \mathrm{OH}), 3.86(\mathrm{~m}, 1 \mathrm{H})$, $5.02(\mathrm{~m}, 1 \mathrm{H}), 7.29(\mathrm{~m}, 2 \mathrm{H}), 7.39(\mathrm{~m}, 2 \mathrm{H}), 7.53(\mathrm{~m}, 2 \mathrm{H}), 7.65(\mathrm{~m}, 1 \mathrm{H}), 8.02(\mathrm{~d}, \mathrm{~J}=7.5 \mathrm{~Hz}, 2 \mathrm{H})$ ppm. ${ }^{13} \mathrm{C}$ NMR $\left(125 \mathrm{MHz}, \mathrm{CDCl}_{3}, 25^{\circ} \mathrm{C}\right): \delta=12.4,26.7,45.8,51.8,127.3,127.5,128.4,128.9$, 129.5, 137.5, 139.8, 141.2, 201.5 ppm. Anal. Calcd for $\mathrm{C}_{17} \mathrm{H}_{18} \mathrm{O}_{2} \mathrm{~S}: \mathrm{C}, 71.32 ; \mathrm{H}, 6.29$; S, 11.18; found: C, 71.35; H, 6.31; S, 11.17.

3-Hydroxy-2-methyl-3-(5-methylthiophen-2-yl)-1-phenylpropan-1-one (3cc). A yellowish oil, IR (neat): $v\left(\mathrm{~cm}^{-1}\right): 3487,3070,2925,1676,1595,1450$. Data for major isomer. ${ }^{1} \mathrm{H}$ NMR $(500$ $\left.\mathrm{MHz}, \mathrm{CDCl}_{3}, 25^{\circ} \mathrm{C}\right): \delta=1.21(\mathrm{~d}, \mathrm{~J}=7.2 \mathrm{~Hz}, 3 \mathrm{H}), 2.50(\mathrm{~s}, 3 \mathrm{H}), 3.18(\mathrm{~d}, \mathrm{~J}=5.0 \mathrm{~Hz}, \mathrm{OH}), 3.91(\mathrm{~m}$, $1 \mathrm{H}), 5.21(\mathrm{~m}, 1 \mathrm{H}), 6.64(\mathrm{~m}, 1 \mathrm{H}), 6.86(\mathrm{~d}, \mathrm{~J}=3.5 \mathrm{~Hz}, 1 \mathrm{H}), 7.51(\mathrm{~m}, 2 \mathrm{H}), 7.62(\mathrm{~m}, 1 \mathrm{H}), 8.05(\mathrm{~d}, \mathrm{~J}$ $=7.4 \mathrm{~Hz}, 2 \mathrm{H}) \mathrm{ppm} .{ }^{13} \mathrm{C} \mathrm{NMR}\left(125 \mathrm{MHz}, \mathrm{CDCl}_{3}, 25^{\circ} \mathrm{C}\right): \delta=15.9,45.1,59.6,71.2,110.4,124.6$, 127.8, 129.1, 129.7, 133.7, 136.9, 154.5, $202.2 \mathrm{ppm}$. Data for minor isomer. ${ }^{1} \mathrm{H}$ NMR $(500 \mathrm{MHz}$, $\left.\mathrm{CDCl}_{3}, 25^{\circ} \mathrm{C}\right): \delta=1.38(\mathrm{~d}, \mathrm{~J}=7.2 \mathrm{~Hz}, 3 \mathrm{H}), 2.49(\mathrm{~s}, 3 \mathrm{H}), 3.41(\mathrm{~d}, \mathrm{~J}=2.7 \mathrm{~Hz}, \mathrm{OH}), 3.87(\mathrm{~m}, 1 \mathrm{H})$, $5.41(\mathrm{~m}, 1 \mathrm{H}), 6.64(\mathrm{~m}, 1 \mathrm{H}), 6.82(\mathrm{~d}, \mathrm{~J}=3.5 \mathrm{~Hz}, 1 \mathrm{H}), 7.55(\mathrm{~m}, 2 \mathrm{H}), 7.64(\mathrm{~m}, 1 \mathrm{H}), 7.99(\mathrm{~d}, \mathrm{~J}=7.7$ $\mathrm{Hz}, 2 \mathrm{H}) \mathrm{ppm} .{ }^{13} \mathrm{C} \mathrm{NMR}\left(125 \mathrm{MHz}, \mathrm{CDCl}_{3}, 25^{\circ} \mathrm{C}\right): \delta=11.9,44.6,49.1,68.6,108.5,123.7,127.2$, 128.9, 129.5, 133.1, 135.4, 151.6, 201.2 ppm. Anal. Calcd for $\mathrm{C}_{15} \mathrm{H}_{16} \mathrm{O}_{2} \mathrm{~S}: \mathrm{C}, 69.23 ; \mathrm{H}, 6.15 ; \mathrm{S}$, 12.30; found: C, 69.25; H, 6.17; S, 12.32 .

3-Hydroxy-2-methyl-3-(5-bromothiophen-2-yl)-1-phenylpropan-1-one (3dc). A yellowish oil, IR (neat): $v\left(\mathrm{~cm}^{-1}\right): 3452,3070,2927,1674,1595,1448$. Data for major isomer. ${ }^{1} \mathrm{H}$ NMR $\left(500 \mathrm{MHz}, \mathrm{CDCl}_{3}, 25^{\circ} \mathrm{C}\right): \delta=1.26(\mathrm{~d}, \mathrm{~J}=7.2 \mathrm{~Hz}, 3 \mathrm{H}), 3.48(\mathrm{~d}, \mathrm{~J}=5.6 \mathrm{~Hz}, \mathrm{OH}), 3.89(\mathrm{~m}, 1 \mathrm{H})$, $5.21(\mathrm{~m}, 1 \mathrm{H}), 6.81(\mathrm{~d}, \mathrm{~J}=3.7 \mathrm{~Hz}, 1 \mathrm{H}), 6.94(\mathrm{~d}, \mathrm{~J}=3.7 \mathrm{~Hz}, 1 \mathrm{H}), 7.56(\mathrm{~m}, 2 \mathrm{H}), 7.67(\mathrm{~m}, 1 \mathrm{H}), 8.02$ $(\mathrm{d}, \mathrm{J}=7.5 \mathrm{~Hz}, 2 \mathrm{H}) \mathrm{ppm} .{ }^{13} \mathrm{C} \mathrm{NMR}\left(125 \mathrm{MHz}, \mathrm{CDCl}_{3}, 25^{\circ} \mathrm{C}\right): \delta=16.2,48.1,67.5,112.4,125.4$, 128.9, 129.3, 129.9, 134.2, 136.6, 148.5, $205.2 \mathrm{ppm}$. Data for minor isomer. ${ }^{1} \mathrm{H}$ NMR (500 MHz, $\left.\mathrm{CDCl}_{3}, 25^{\circ} \mathrm{C}\right): \delta=1.36(\mathrm{~d}, \mathrm{~J}=7.2 \mathrm{~Hz}, 3 \mathrm{H}), 3.67(\mathrm{~d}, \mathrm{~J}=2.5 \mathrm{~Hz}, \mathrm{OH}), 3.79(\mathrm{~m}, 1 \mathrm{H}), 5.43(\mathrm{~m}, 1 \mathrm{H})$, $6.78(\mathrm{~d}, \mathrm{~J}=3.7 \mathrm{~Hz}, 1 \mathrm{H}), 6.96(\mathrm{~d}, \mathrm{~J}=3.7 \mathrm{~Hz}, 1 \mathrm{H}), 7.52(\mathrm{~m}, 2 \mathrm{H}), 7.63(\mathrm{~m}, 1 \mathrm{H}), 7.99(\mathrm{~d}, \mathrm{~J}=7.8$ $\mathrm{Hz}, 2 \mathrm{H}) \mathrm{ppm} .{ }^{13} \mathrm{C} \mathrm{NMR}\left(125 \mathrm{MHz}, \mathrm{CDCl}_{3}, 25^{\circ} \mathrm{C}\right): \delta=12.5,53.8,70.9,111.5,124.1,128.9$, 129.2, 129.8, 134.0, 135.8, 147.9, 204.8 ppm. Anal. Calcd for $\mathrm{C}_{14} \mathrm{H}_{13} \mathrm{O}_{2} \mathrm{SBr}$ : C, 51.69; H, 4.00; S, 9.84; found: C, 51.70; H, 3.98; S, 9.85 .

3-Hydroxy-2-methyl-3-(3-methylthiophen-2-yl)-1-phenylpropan-1-one (3ec). A colorless oil, IR (neat): $v\left(\mathrm{~cm}^{-1}\right): 3465,3062,2925,1674,1595,1450$. Data for major isomer. ${ }^{1} \mathrm{H}$ NMR $(500$ $\left.\mathrm{MHz}, \mathrm{CDCl}_{3}, 25^{\circ} \mathrm{C}\right): \delta=1.12(\mathrm{~d}, \mathrm{~J}=7.2 \mathrm{~Hz}, 3 \mathrm{H}), 2.26(\mathrm{~s}, 3 \mathrm{H}), 2.87(\mathrm{~d}, \mathrm{~J}=3.8 \mathrm{~Hz}, \mathrm{OH}), 3.93(\mathrm{~m}$, $1 \mathrm{H}), 5.45(\mathrm{~m}, 1 \mathrm{H}), 6.86(\mathrm{~d}, \mathrm{~J}=5.0 \mathrm{~Hz}, 1 \mathrm{H}), 7.27(\mathrm{~d}, \mathrm{~J}=5.0 \mathrm{~Hz}, 1 \mathrm{H}), 7.49(\mathrm{~m}, 2 \mathrm{H}), 7.61(\mathrm{~m}, 1 \mathrm{H})$, $8.07(\mathrm{~d}, \mathrm{~J}=7.4 \mathrm{~Hz}, 2 \mathrm{H}) \mathrm{ppm} .{ }^{13} \mathrm{C} \mathrm{NMR}\left(125 \mathrm{MHz}, \mathrm{CDCl}_{3}, 25^{\circ} \mathrm{C}\right): \delta=15.4,45.7,57.5,68.2$, 
114.5, 124.6, 127.6, 128.9, 129.4, 133.9, 135.8, 153.1, $202.0 \mathrm{ppm}$. Data for minor isomer. ${ }^{1} \mathrm{H}$ NMR (500 MHz, $\left.\mathrm{CDCl}_{3}, 25^{\circ} \mathrm{C}\right): \delta=1.42(\mathrm{~d}, \mathrm{~J}=7.2 \mathrm{~Hz}, 3 \mathrm{H}), 2.34(\mathrm{~s}, 3 \mathrm{H}), 3.28(\mathrm{~d}, \mathrm{~J}=2.4 \mathrm{~Hz}$, $\mathrm{OH}), 3.85(\mathrm{~m}, 1 \mathrm{H}), 5.50(\mathrm{~m}, 1 \mathrm{H}), 6.80(\mathrm{~d}, \mathrm{~J}=5.0 \mathrm{~Hz}, 1 \mathrm{H}), 7.17(\mathrm{~d}, \mathrm{~J}=5.0 \mathrm{~Hz}, 1 \mathrm{H}), 7.55(\mathrm{~m}$, 2H), $7.65(\mathrm{~m}, 1 \mathrm{H}), 7.99(\mathrm{~d}, \mathrm{~J}=7.9 \mathrm{~Hz}, 2 \mathrm{H}) \mathrm{ppm} .{ }^{13} \mathrm{C} \mathrm{NMR}\left(125 \mathrm{MHz}, \mathrm{CDCl}_{3}, 25^{\circ} \mathrm{C}\right): \delta=13.9$, 42.6, 49.5, 63.6, 113.2, 124.6, 127.1, 128.9, 129.1, 133.3, 134.5, 151.0, 201.1 ppm. Anal. Calcd for $\mathrm{C}_{15} \mathrm{H}_{16} \mathrm{O}_{2} \mathrm{~S}: \mathrm{C}, 69.23 ; \mathrm{H}, 6.15 ; \mathrm{S}, 12.30$; found: $\mathrm{C}, 69.25 ; \mathrm{H}, 6.17 ; \mathrm{S}, 12.32$.

3-(Furan-2-yl)-3-hydroxy-2-methyl-1-phenylpropan-1-one (3fc). A yellowish oil, IR (neat): $v$ $\left(\mathrm{cm}^{-1}\right): 3450,3075,2930,1674,1592,1455$. Data for major isomer. ${ }^{1} \mathrm{H}$ NMR $\left(500 \mathrm{MHz}, \mathrm{CDCl}_{3}\right.$, $\left.25^{\circ} \mathrm{C}\right): \delta=1.35(\mathrm{~d}, \mathrm{~J}=7.2 \mathrm{~Hz}, 3 \mathrm{H}), 3.31(\mathrm{~d}, \mathrm{~J}=3.4 \mathrm{~Hz}, \mathrm{OH}), 4.15(\mathrm{~m}, 1 \mathrm{H}), 5.08(\mathrm{~m}, 1 \mathrm{H}), 6.37$ $(\mathrm{m}, 2 \mathrm{H}), 7.41(\mathrm{~m}, 1 \mathrm{H}), 7.51(\mathrm{~m}, 2 \mathrm{H}), 7.66(\mathrm{~m}, 1 \mathrm{H}), 8.04(\mathrm{~m}, 2 \mathrm{H}) \mathrm{ppm} .{ }^{13} \mathrm{C} \mathrm{NMR}(125 \mathrm{MHz}$, $\left.\mathrm{CDCl}_{3}, 25^{\circ} \mathrm{C}\right): \delta=11.8,47.5,49.2,109.2,109.3,128.7,128.8,129.1,132.3,136.8,143.8,202.9$ ppm. Data for minor isomer. ${ }^{1} \mathrm{H} \mathrm{NMR}\left(500 \mathrm{MHz}, \mathrm{CDCl}_{3}, 25^{\circ} \mathrm{C}\right): \delta=1.22(\mathrm{~d}, \mathrm{~J}=7.2 \mathrm{~Hz}, 3 \mathrm{H})$, $3.24(\mathrm{~d}, \mathrm{~J}=5.8 \mathrm{~Hz}, \mathrm{OH}), 4.01(\mathrm{~m}, 1 \mathrm{H}), 5.27(\mathrm{~m}, 1 \mathrm{H}), 6.39(\mathrm{~m}, 2 \mathrm{H}), 7.39(\mathrm{~m}, 1 \mathrm{H}), 7.55(\mathrm{~m}, 2 \mathrm{H})$, $7.62(\mathrm{~m}, 1 \mathrm{H}), 7.99(\mathrm{~m}, 2 \mathrm{H}) \mathrm{ppm} .{ }^{13} \mathrm{C} \mathrm{NMR}\left(125 \mathrm{MHz}, \mathrm{CDCl}_{3}, 25^{\circ} \mathrm{C}\right): \delta=11.2,46.4,50.1,110.5$, 110.7, 128.0, 128.5, 128.6, 134.3, 137.9, 141.4, 201.9 ppm. Anal. Calcd for $\mathrm{C}_{14} \mathrm{H}_{14} \mathrm{O}_{3}: \mathrm{C}, 73.04$; H, 6.08; found: C, 73.07; H, 6.11 .

\section{Acknowledgements}

The authors are grateful to the research council of Guilan University for the partial support of this study.

\section{References}

1. Meijere, A.; Diederich, F. Metal-Catalyzed Cross-Coupling Reactions, $2^{\text {nd }}$ Edn.; WileyVCH: New York, 2004; Vol. 2.

2. Sakthivel, K.; Notz, W.; Bui, T.; Barbas, III, C. F. J. Am. Chem. Soc. 2001, 123, 5260.

3. Mukaiyama, T. Angew. Chem. Int. Ed. 2004, 43, 5590.

4. Machajewski, T. D.; Wong, C.-H. Angew. Chem. Int. Ed. 2000, 39, 1352.

5. March, J. Advanced Organic Chemistry: Reactions, Mechanisms and Structure, $4^{\text {th }}$ Edn.; Wiley-Interscience: New York, 1992.

6. Mukaiyama, T. Org. React. 1982, 28, 203.

7. Trost, B. M.; Oi, S. J. Am. Chem. Soc. 2001, 123, 1230.

8. Slough, G. A.; Bergman, R. G.; Heathcock, C. H. J. Am. Chem. Soc. 1989, 111, 938.

9. Trost, B. M.; Silcoff, E. R.; Ito, H. Org. Lett. 2001, 3, 2497.

10. Murahashi, S.-I.; Takaya, H. Acc. Chem. Res. 2000, 33, 225.

11. Crévisy, C.; Wietrich, M.; Boulaire, V. L.; Uma, R.; Grée, R. Tetrahedron Lett. 2001, 42, 395. 
12. Trost, B. M.; Ito, H. J. Am. Chem. Soc. 2000, 122, 12003.

13. (a) Murahashi, S.-I. Ruthenium in Organic Synthesis, Wiley-VCH: New York, 2004. (b) Bruneau, C.; Dixneuf, P. H. Ruthenium Catalysts and Fine Chemistry, Springer, 2004. (c) Muri, S. Activation of Unreactive Bonds and Organic Synthesis, Springer, 1999. (d) Trost, B. M.; Toste, F. D.; Pinkerton, A. B. Chem. Rev. 2001, 101, 2067.

14. Wei, H.-X.; Jasoni, R. L.; Shao, H.; Hu, J.; Paré, P. W. Tetrahedron 2004, 60, 11829.

15. List, B.; Pojarliev, P.; Castello, C. Org. Lett. 2001, 3, 573.

16. Wei, H.-X.; Li, K.; Zhang, Q.; Jasoni, R. L.; Hu, J.; Paré, P. W. Helv. Chim. Acta 2004, 87, 2354.

17. (a) Tabatabaeian, K.; Mamaghani, M.; Mahmoodi, N. O.; Khorshidi, A. Can. J. Chem. 2006, 84, 1541. (b) Tabatabaeian, K.; Mamaghani, M.; Mahmoodi, N. O.; Khorshidi, A. J. Mol. Catal. A. 2007, 270, 112. (c) Tabatabaeian, K.; Mamaghani, M.; Mahmoodi, N. O.; Khorshidi, A. Catal. Commun. 2008, 9, 416. (d) Tabatabaeian, K.; Mamaghani, M.;Mahmoodi, N. O.; Khorshidi, A. Tetrahedron Lett. 2008, 49, 1450.

18. Stephenson, T. A.; Wilkinson, G. J. Inorg. Nucl. Chem. 1966, 28, 945. 